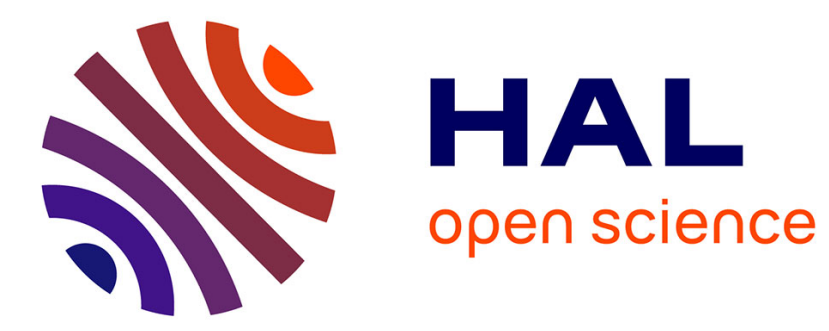

\title{
Volume-Aware Positioning in the Context of a Marine Port Terminal
}

Yesid Jarma, Golnaz Karbaschi, Marcelo Dias de Amorim, Farid Benbadis, Guillaume Chelius

\section{- To cite this version:}

Yesid Jarma, Golnaz Karbaschi, Marcelo Dias de Amorim, Farid Benbadis, Guillaume Chelius. Volume-Aware Positioning in the Context of a Marine Port Terminal. Computer Communications, 2011, 34 (8), pp.962-972. 10.1016/j.comcom.2011.02.003 . hal-00579839

\section{HAL Id: hal-00579839 \\ https://hal.science/hal-00579839}

Submitted on 25 Mar 2011

HAL is a multi-disciplinary open access archive for the deposit and dissemination of scientific research documents, whether they are published or not. The documents may come from teaching and research institutions in France or abroad, or from public or private research centers.
L'archive ouverte pluridisciplinaire HAL, est destinée au dépôt et à la diffusion de documents scientifiques de niveau recherche, publiés ou non, émanant des établissements d'enseignement et de recherche français ou étrangers, des laboratoires publics ou privés. 


\title{
Volume-Aware Positioning in the Context of a Marine Port Terminal ${ }^{\text {th }}$
}

\author{
Yesid Jarma*,a, Golnaz Karbaschi ${ }^{\mathrm{b}}$, Marcelo Dias de Amorimª ${ }^{\mathrm{a}}$, Farid Benbadis ${ }^{\mathrm{c}}$, Guillaume Chelius ${ }^{\mathrm{d}}$ \\ ${ }^{a}$ LIP6/CNRS - UPMC Sorbonne Universités, 4 place Jussieu, 75005 Paris, France \\ ${ }^{b}$ INRIA Saclay, Parc Orsay Université, 4 Rue Jacques Monod, 91893 Orsay Cedex, France \\ ${ }^{c}$ Thales Communications, 160 Boulevard de Valmy, 92704 Colombes Cedex, France \\ ${ }^{d}$ INRIA Rhône-Alpes, 655 avenue de l'Europe, Montbonnot, 38334 Saint Ismier Cedex, France
}

\begin{abstract}
The rapid proliferation of mobile computing devices and local wireless networks over the past few years has promoted a continuously growing interest in location-aware systems and applications. The main problem with existing positioning techniques is that they are designed to position dimensionless objects. Such an assumption may lead to practical inconsistencies, as it results in neglecting the effects of the volume of an object, its physical characteristics, and its spatial arrangement on signal propagation. In this paper, we consider the problem of positioning cargo containers in a marine port terminal, where such characteristics can be finely estimated. Based on the signal propagation map of a container yard, we propose VAPS, a volume-aware positioning system that takes advantage of the waveguide effect generated by the containers. Although VAPS is specific to the marine port scenario, its design principles can be extended and adapted to other situations. VAPS maps discrete RSSI levels into hop-counts and relies on realistic propagation models to obtain near-perfect positioning at a very low control overhead. Our extensive evaluations show how to set the parameters required in the VAPS algorithm. The results demonstrate that, in scenarios where the assumptions made by traditional approaches fail, the considerations of VAPS make the difference.
\end{abstract}

Key words: GPS-free positioning systems, signal propagation, waveguide effect.

\section{Introduction}

Having the coordinates of a node can be of great utility in numerous applications of wireless networks, including object tracking, warehouse inventory, health care, and environmental monitoring, to cite a few. Therefore, positioning systems have been and still are the focus of extensive research. The main approaches in use today rely on satellite-based systems such as GPS [1], Glonass [2], as well as the upcoming Galileo [3] and Beidou [4]. Although straightforward and widely adopted, satellite-based solutions cannot be used in a number of scenarios (e.g., indoor, underwater, and underground deployments) and can involve high economic and energetic costs. There have been many attempts in the literature to provide alternative solutions, commonly referred to as GPS-free positioning systems. These are roughly classified into two classes: signal-based and topology-based methods. In the first category, the strategy most frequently adopted is to measure the distance between the target element and several reference points (whose coordinates are known) based on metrics such as the Received Signal Strength Indicator (RSSI) [5, 6, 7, 8, 9], Time of Arrival (ToA) [10],

\footnotetext{
This document is an extended version of our previous paper "VAPS: Positioning With Spatial Constraints", presented at IEEE WoWMoM 2009.

${ }^{*}$ Corresponding author

Email addresses: yesid. jarma@lip6.fr (Yesid Jarma), golnaz . karbaschi@inria.fr (Golnaz Karbaschi), marcelo.amorim@lip6.fr (Marcelo Dias de Amorim), farid.benbadis@fr.thalesgroup.com (Farid Benbadis), guillaume.chelius@inria.fr (Guillaume Chelius)
}

Time Difference of Arrival (TDoA) [11], or Angle of Arrival (AoA) [12]. In the second category, the traditional approach is to determine the distance of a node to a number of reference points by using network topology metrics such as the hopcount $[13,14,15,16]$. In both methods, the coordinates of the target point are inferred through multi-lateration or similar techniques.

In this paper, we focus on a class of deployment scenarios where the abovementioned solutions cannot be used. In fact, existing methods are designed to position dimensionless nodes without considering their volume, physical composition, or any other spatial constraints. The implicit assumption is that the node to be positioned is a point in the space. This is a potential source of errors because the volume, the physical characteristics of the objects to position, and their spatial arrangement may have a major impact on the signal propagation. The particular characteristics of large objects may lead to new constraints such as a strong attenuation or reflection of propagated signals. In some cases, as the marine port scenario considered in this paper, objects have metallic surfaces that lead to high reflection and blocking effects on the transmitted signals. Therefore, certain arrangements of metallic objects may cause waveguide effects, inducing homogeneous signal propagation throughout the area. Uniform propagation of signals may lead to ambiguity in determining the positions of the objects, as the signal attenuation becomes completely different from the one observed in open spaces.

To our knowledge, there is no work in the literature deal- 


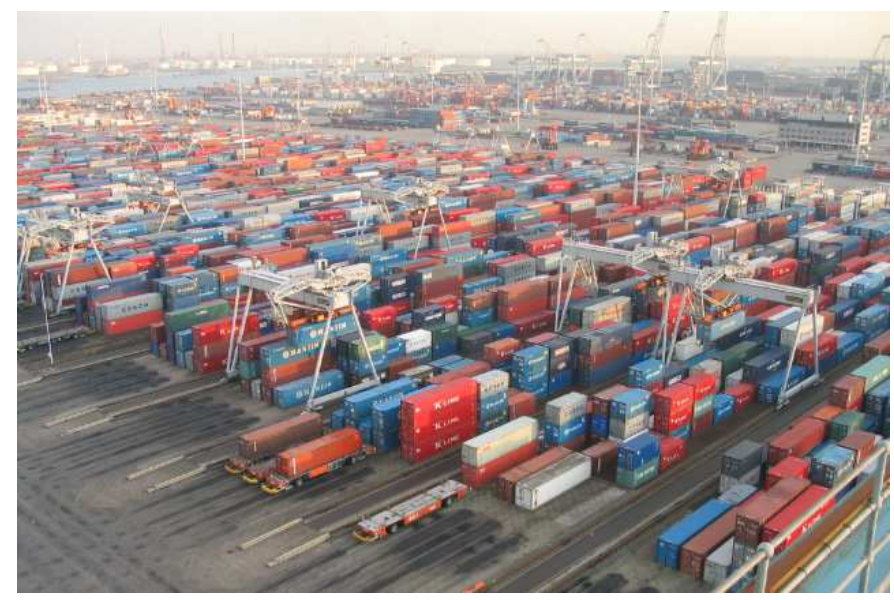

Figure 1: Container yard in a marine port terminal.

ing with such specific challenges. In order to effectively bridge this gap, we propose VAPS, a Volume Aware Positioning System to position large metallic objects. Contrarily to traditional solutions, VAPS brings the following innovative features:

- Signal propagation-aware positioning. VAPS takes into account and benefits from the waveguide and blocking effects caused by metallic objects. This is fundamental in our scenario as these effects completely compromise the correlation between distance and reference parameters such as signal strength, propagation time, and angle of arrival. For this reason, in our scenario, traditional systems are useless as they rely on such correlations when computing coordinates.

- Dimension-aware positioning. VAPS falls under the umbrella of hop-count-based systems; however, since containers have well-defined dimensions, hop-counts can be directly translated into distances. Furthermore, VAPS is able to correct possible erroneous coordinates, therefore preventing physically impossible situations such as the overlap of two or more elements.

Taking spatial constraints into account is of course scenariodependent. In this paper, we specifically refer to a reservoir of cargo containers at a marine port terminal arranged along long rows, several containers high, in a limited space. The containers are metallic, inducing high reflection, which leads to distinct waveguide effects. This means that signal attenuation in the space separating two rows of containers is almost null. By applying realistic signal propagation that we obtained through extensive simulations, we show that: (i) VAPS works where traditional solutions are unfeasible (ii) VAPS leads to highly accurate positioning, and (iii) VAPS is energy-efficient by generating very low control overhead.

The remainder of this paper is structured as follows. In Section 2, we describe the context of the paper, and in Section 3 we illustrate the particularities of signal propagation in positioning metallic objects with large dimensions. In the same section, we clearly state the problem of using traditional methods

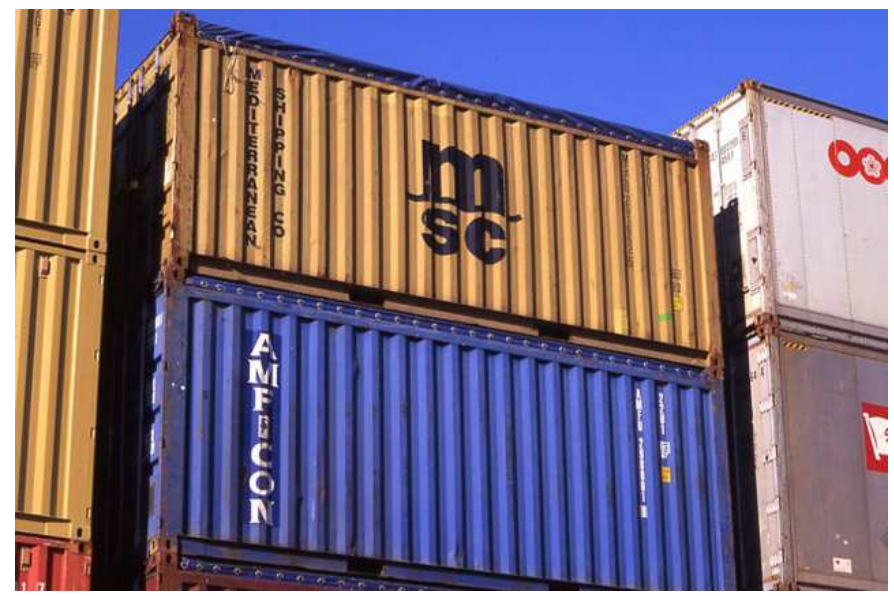

Figure 2: Stacks of cargo containers in a marine port terminal.

when attempting to position metallic objects with large dimensions. Section 4 presents the VAPS algorithm for positioning cargo containers, while Section 5 evaluates our algorithm via simulations. In Section 6 we discuss the performance of our algorithm and give insights on remaining open issues. Section 7 gives an overview on prior works in positioning algorithms for multi-hop wireless networks. Finally, we conclude the paper in Section 8.

\section{Context}

During the latest decade, the world has been shaped by the influence of globalization. To sustain economic growth, many companies have started to develop new markets outside their home countries. Therefore, international transportation systems became under increasing pressures to support additional demands. The introduction of cargo containers resulted in considerable improvements in efficiency, lowering costs, and boosting global trade flows.

The target scenario considered in this work is the localization of cargo containers in a marine port terminal. ${ }^{1}$ Many modern marine ports are used as intermediate destinations where the cargo is changed form one transportation method to another. In large ports, such as the one depicted in Fig. 1, cargo container facilities may extend over 170 hectares, where several thousand containers are held and moved every day. Clearly, locating and tracking such a large number of containers require complex logistics.

When cargo containers are being held in the port, they are usually stacked one on top of another, with little vertical space between them (about $10 \mathrm{~cm}$ ), and narrow aisles (about $1 \mathrm{~m}$ ) separating two rows of stacks (see Fig. 2). A row of stacks may be up to 100 meters long, and 20 meters high. This particular arrangement and the metallic composition of cargo containers both have an impact on the propagation of signals between two

${ }^{1}$ More specifically, this scenario is the one addressed in the context of the French project "Watch and Warn" in collaboration with the Le Havre harbor [17]. 


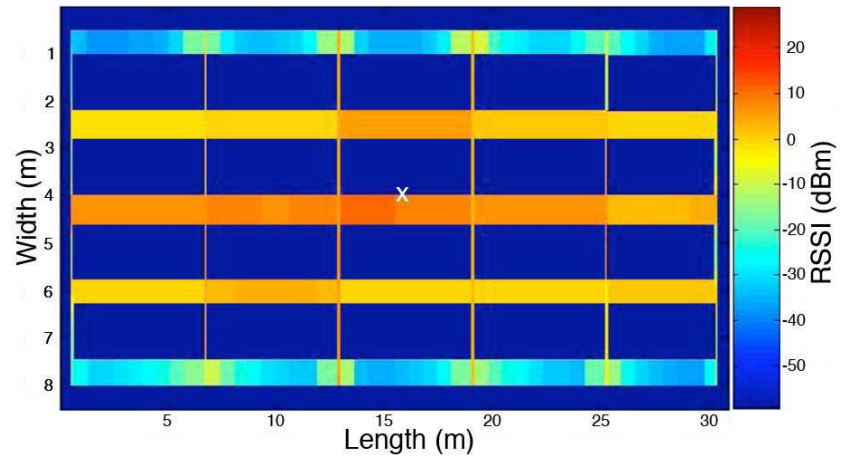

(a) Above view

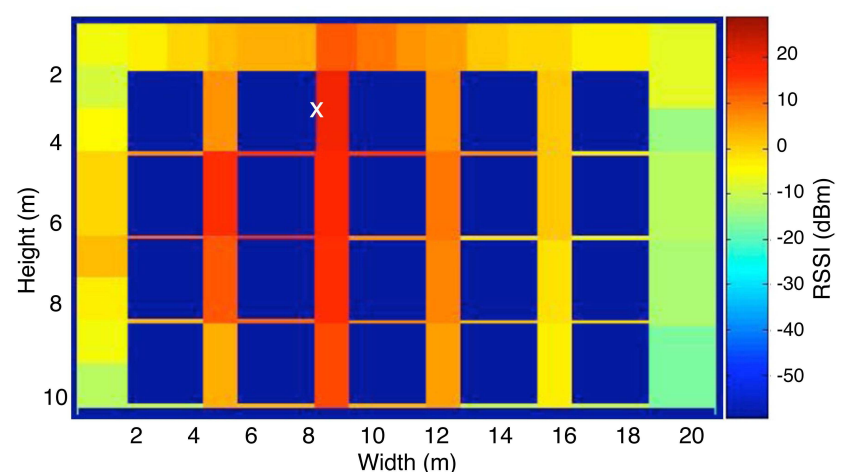

(b) Transversal view.

Figure 3: Signal propagation of a sensor in a field of rectangular metallic objects (dark rectangles represent containers). A white "X" represents the transmitting sensor.

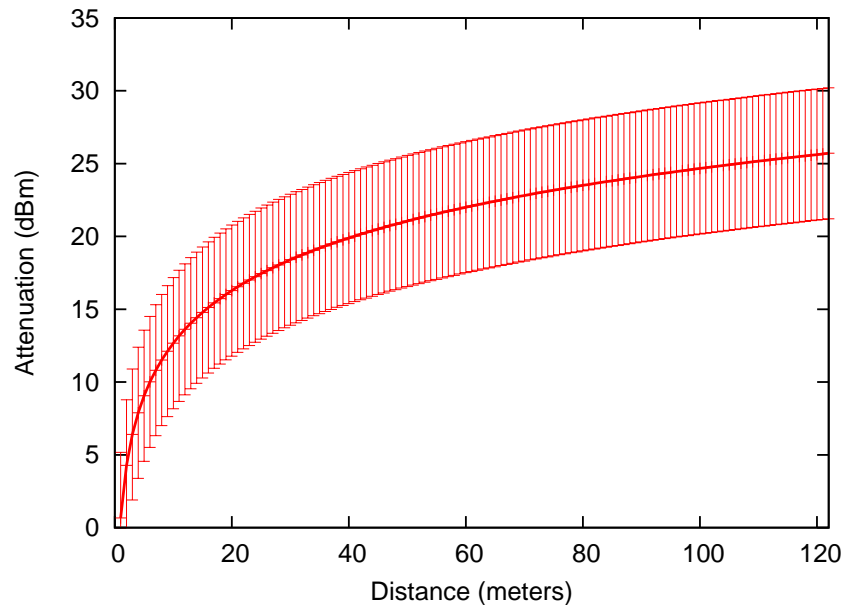

(a) Attenuation in an aisle between two stacks of containers.

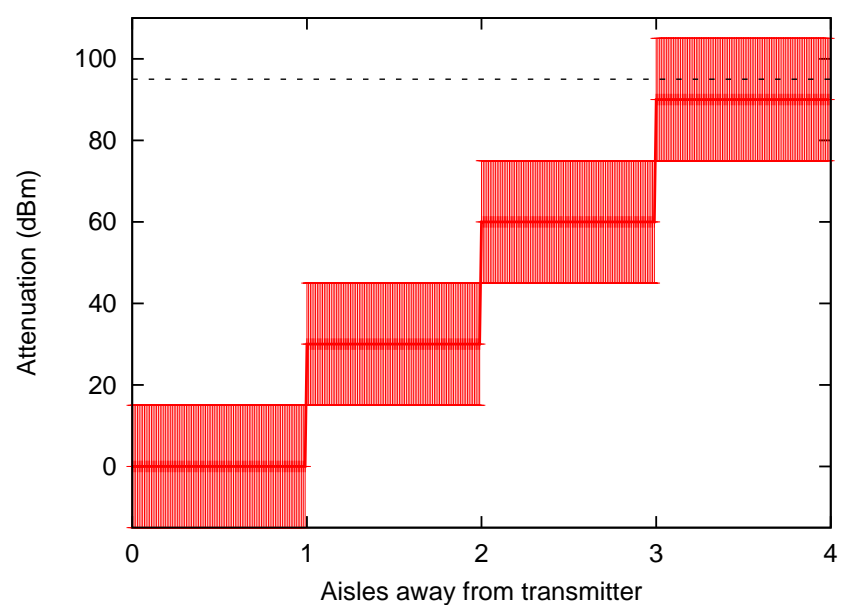

(b) Attenuation from one aisle to another.

Figure 4: Observed Path Loss Model. Vertical error bars represent the tolerance.

rows of stacks of containers. These particularities are described and analyzed in the following section.

\section{Problem Statement}

\subsection{Analyzing the waveguide effect}

To better assess the effects of the particular arrangement and characteristics of these objects on wave propagation, we conducted a set of precise radio propagation simulations. To this end, the WILDE (WIreless LAN DEsign) tool was used [18, 19]. Fig. 3 illustrates the obtained results. In these figures, dark rectangles represent the containers disposed in rows; one wireless sensor (placed on the longest surface of a container and represented with the white ' $\mathrm{X}$ ' in both figures) sends a signal whose RSSI is measured in the rest of the space. The RSSI is represented by colors. In the aisles where the signal is transmitted, the color is something between red and orange, meaning that the signals are received with almost the same power-level they were transmitted. In the following aisles, where the colors are yellow, green, or blue, the received signals are attenuated. This attenuation, however, happens sharply, and the power level remains almost constant all along the same aisle (except for the border effect at crossing points).

In these results, two particular phenomena can be observed. First, wave propagation in the aisles between two rows of stacks of containers closely resembles a waveguide. Nevertheless, it is not a tuned waveguide and therefore path loss occurs. Fig. 4(a) shows the observed path loss model for an aisle between two rows of stacks of containers. Error bars represent an error margin of $\pm 5 \mathrm{dBm}$. Since the attenuation inside an aisle is well below the sensitivity threshold, any node transmitting inside an aisle can be heard several hundred meters away. Clearly, the observed model greatly differs from the traditional free-space path loss model, where the typical range is around 50 meters.

The second observed phenomenon is that the objects act as barriers. The metallic nature of cargo containers and their size prevent the signals from reaching near points. This behavior is illustrated in Fig. 4(b). Error bars represent an error margin of $\pm 15 \mathrm{dBm}$. Even if two adjacent aisles between containers are a few meters away from each other, the attenuation changes abruptly from one aisle to the next. Nevertheless, all along one aisle, wave propagation continues to follow the waveguide-like 


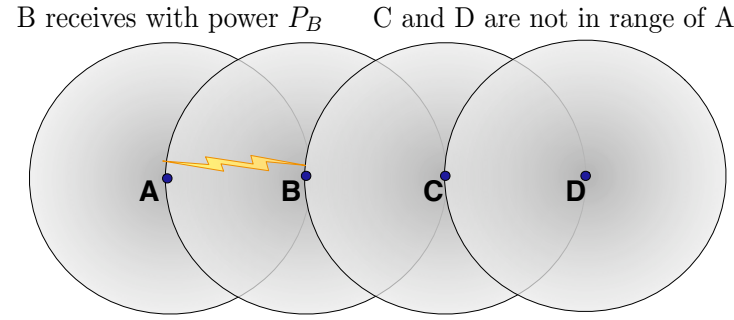

$\mathrm{B}$ distance estimate is $d\left(P_{B}\right) \quad \mathrm{C}$ and $\mathrm{D}$ can't estimate distance to A

(a) In open space, node B is in range of node $\mathrm{A}$ and can estimate its distance to it. Nodes C and D are outside node A's range.
B, $\mathrm{C}$ and $\mathrm{D}$ receive with powers $P_{B} \approx P_{C} \approx P_{D}$

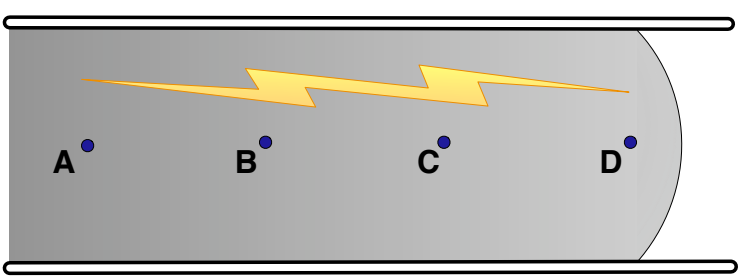

$\mathrm{B}, \mathrm{C}$ and $\mathrm{D}$ distance estimates are $d\left(P_{B}\right) \approx d\left(P_{C}\right) \approx d\left(P_{D}\right)$

(b) Inside a waveguide, nodes $\mathrm{B}, \mathrm{C}$ and $\mathrm{D}$ are in range of node $\mathrm{A}$ and can estimate its distance to it. However, because of homogeneous signal propagation they will estimate they are closer than they actually are.

Figure 5: Distance estimation using RSSI in open space and inside a waveguide.

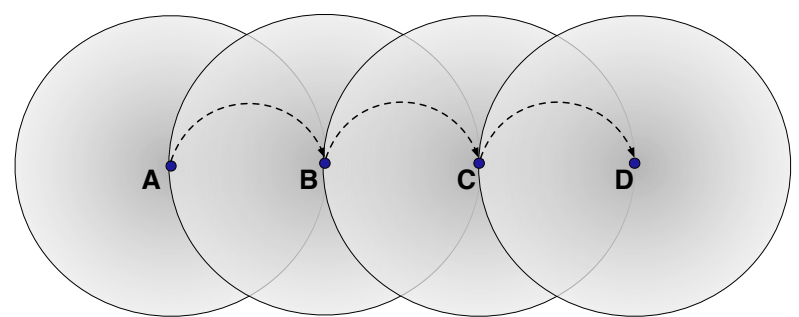

(a) In open space, node A communicates with nodes C and D in a hopby-hop way.

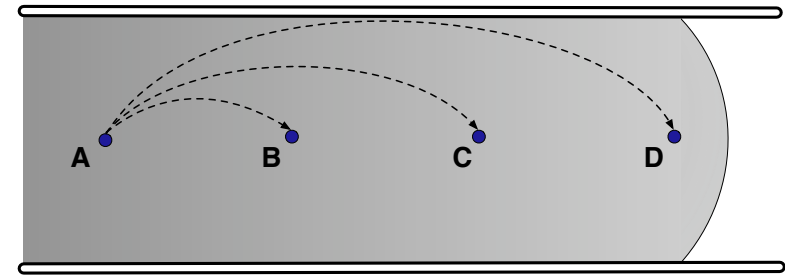

(b) Inside a waveguide, node A can communicate directly with nodes $\mathrm{C}$ and D.

Figure 6: Distance estimation counting number of hops in open space and inside a waveguide.

path loss model observed in Fig. 4(a). Therefore, the attenuation is almost homogeneous along an aisle.

\subsection{Limitations of existing approaches}

To our knowledge, all existing positioning methods assume that objects are just small points in space, without any dimensions whatsoever. Moreover, there is no consideration of the effects that the volume and physical characteristics of an object may have on signal propagation. Therefore, given the particular characteristics of wave propagation in the previously presented context, any attempt to position large metallic objects using existing techniques will lead to considerable positioning errors. To better illustrate this, we analyze the issues that rise when two of the most popular positioning techniques are used to position objects inside a waveguide. Further details on existing techniques are presented in Section 7.

One of the most used techniques consists in estimating the distance between an object and at least three reference points whose positions are known. Then, by trilateration, the object calculates its position relatively to the positions of the reference points. The distance between two nodes can be calculated using for example the RSSI. If both nodes are identical (i.e., they transmit with the same power and they have the same sensitivity threshold) the receiving node can estimate its distance to the transmitting node by using the RSSI and a path loss model. In Fig. 5(a), we represent a topology with four nodes. Here, node $\mathrm{B}$ is the unique node under the coverage range of node $\mathrm{A}$, and therefore it can accurately estimate its distance to node A by using RSSI and a free-space path loss model [20]. Since nodes
$\mathrm{C}$ and D are outside A's range, they cannot estimate their distance. In contrast to a free-space model, inside a waveguide (see Fig. 5(b)), nodes B, C, and D are all in the communication range of node $\mathrm{A}$, and can thus estimate their distance to it. However, because of the particularities of signal propagation inside a waveguide, all four nodes will receive the transmitted signal with almost the same power. By using a free-space path loss model, they will estimate their positions to be closer than they actually are.

Another technique consists in measuring the distance from a node to a reference point in number of hops. This information can be then used to calculate a distance in meters, or directly as coordinates. In Fig. 6(a), nodes A, B, C, and D communicate with each other in a hop-by-hop fashion. In this case, node D is three hops away from node $\mathrm{A}$. Inside a waveguide, node A can communicate directly with nodes B, C, and D. In such a case, the use of a hop-count technique attributes the same coordinates to those three nodes, although they are placed at significantly different distances from node A.

\section{VAPS: Volume-Aware Positioning System}

To address the new challenges in positioning with spatial constraints, we adopt a strategy that differs from those of traditional approaches. We mainly undertake two actions. First, every object should be equipped with more than one communicating device (or sensor) so that we can integrate the notion of dimension. We assume that the sensors on the same object are wired to each other and exchange positioning information be- 


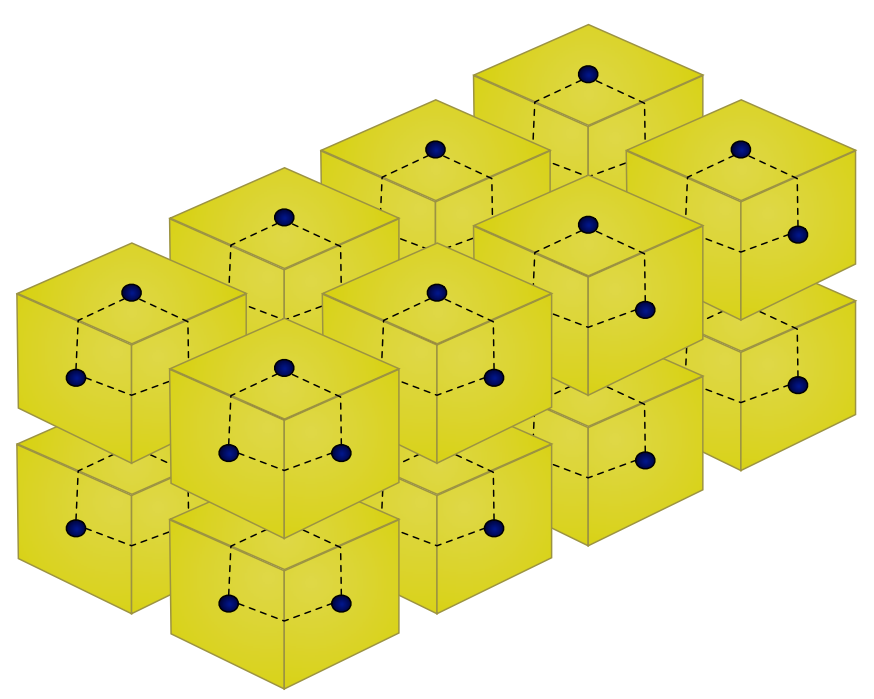

Figure 7: This figure shows a grid of 16 objects with three sensors each to enable three dimensional positioning. The sensors are represented by blue circles, and are wired to each others. Note that all sensors are facing the same directions.

tween them instantly, and without consuming any wireless resource. In the description of the algorithm, we call these nodes corresponding nodes. Fig. 7 shows an example of node equipment for three dimensional positioning. In this particular example, each object is equipped with three nodes (one for each dimension) wired to each others. Note that all nodes are on the exact same surface on each object and therefore they all have the same direction. Second, we consider in the design of our algorithm the effects that metallic objects may have on signal propagation, namely the waveguide effect. In this section, we first specify the preliminaries of our approach. The details of VAPS come thereafter.

\subsection{Determining $N$ discrete distinguishable power levels}

Without loss of generality, we assume that all sensor nodes have the same transmission power $P_{r}$ and the same sensitivity threshold. As a consequence of the waveguide effect, signal propagation in an aisle is almost homogeneous; therefore a transmitting node is heard with almost the same reception power all along the aisle it is placed in. Using this particular phenomenon, we can easily conclude that every node hearing the maximum received power level are estimated to be in the same aisle as the transmitting node. The transmitted signal will propagate throughout the area and it will be heard in the adjacent aisles, but with an attenuated power level. As observed in Fig. 3, the attenuation happens sharply. This attenuation, however, follows the waveguide effect and thus remains almost constant all along the same aisle.

We assume that, in general, a transmission originated inside any aisle can be heard at the next $N$ consecutive aisles. Using the particular waveguide/blocking effects of this context, we are able to define $N$ discrete and clearly distinguishable power levels. The number of power levels is determined by the number of aisles away from the transmitting node where the RSSI is

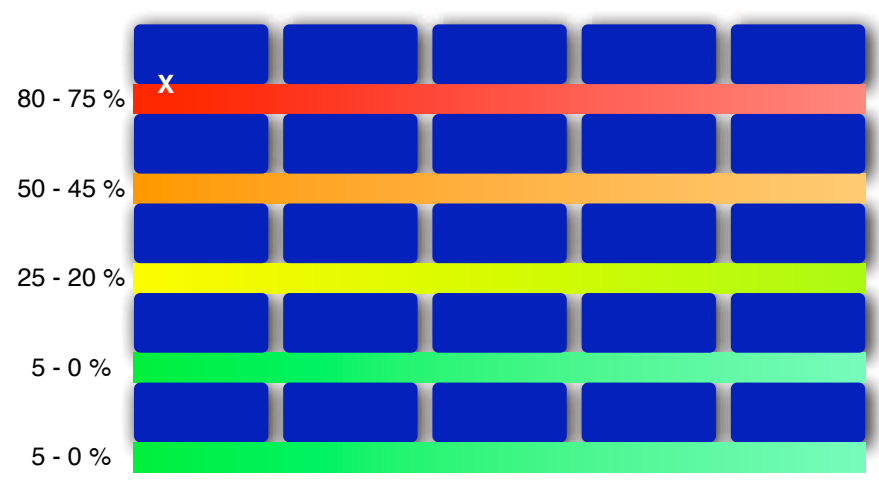

Figure 8: This figure shows a situation where X's signal is easily distinguishable from an aisle to another till the third aisle. The received signal at the forth and fifth aisle is just below the sensitivity threshold of the sensors.

above the sensitivity threshold of the nodes. To better illustrate this, we take as example the topology depicted in Fig. 8.

In this particular case, there are 25 rectangular metallic objects arranged in a $5 \times 5$ grid. We set a transmitting node, represented with a white " $X$ ", in the lower side of the container located at the top left corner of the topology. Other nodes (not depicted in the figure) located in the lower sides of the rest of the containers measure the power of the received signal. In this example, we assume that the sensitivity threshold is $20 \%$ of the original power. When the transmitting node sends a message, the nodes located on the same aisle as the transmitter receive the signal with $80-75 \%$ of its transmitting power. Nodes located one aisle away will receive the signal with $50-45 \%$ of its original power, while nodes two aisles away will receive the signal with $25-20 \%$ of its original power. For the nodes located three and four aisles away, the received signal will be just below their sensitivity threshold. Nodes in the first three aisles can easily detect the signal, and the receiving power from one aisle to the next one is clearly distinguishable. On the other hand, there is no clear distinction between the power received in the fourth and fifth aisles, and in some cases, a number of nodes will not be able to detect the transmission. In this particular example, $N$ is thus set to 3 . It means that one transmission in an aisle is able to determine the aisle number of objects of three aisles.

The value of $N$ depends on several factors, including the reception sensibility of the sensors, the width of the aisles, possible changes in the propagation characteristics of the wireless medium, and the transmission power $P_{r}$. Note that the value of $N$ can be different across each direction. In the example depicted in Fig. 8, horizontal aisles are wider than vertical aisles, and consequently the waveguide effect in the former is more important than in the latter. Therefore, in the general case, we consider $N_{x}$ and $N_{y}$ as different values for each direction.

\subsection{Description of the algorithm}

For the sake of presentation, we consider a two-dimensional space with two axes $\mathrm{X}$ and $\mathrm{Y}$. Nevertheless, extending the al- 
gorithm to three dimensions is straightforward. ${ }^{2}$ Let $L_{i}$ be the power level measured at aisle $i$ from the emitter and $V=\left\{L_{1}, \ldots, L_{N}\right\}$ be the set of these measurements ( $i=1$ means the same aisle of the emitter). An object (i.e., a container) has two sensors placed on orthogonal surfaces. We refer to these sensors as corresponding nodes and call them $x$-node and $y$-node respectively to the axis they contribute to. The objects are assumed to have a direction, such that $x$-nodes and $y$-nodes are placed on the same surface on each one of the containers. We compute coordinates with regard to an object placed in a corner of the topology whose coordinates are, by definition, $(X=0, Y=0)$. We refer to this particular container as the anchor. Note that we need only one anchor.

The anchor starts the positioning process by broadcasting a message toward the $\mathrm{x}$-axis (via its $x$-node). To prevent possible collisions that may interfere with positioning, we introduce a back-off time when broadcasting toward the y-axis (via its $y$ node). Messages broadcasted toward the $\mathrm{x}$-axis determine the column number and toward the $y$-axis the row number. Each message contains two pieces of information: a coordinate $(X$ or $Y$ ), and the axis it corresponds to. A node that receives a message analyzes the axis information to determine whether it should process the message or not: $x$-nodes only process $\mathrm{x}$-axis messages while $y$-nodes only process $y$-axis messages. If the message is processed, the positioning information together with RSSI information is analyzed. If the message is received with a RSSI that is within the range $\left[L_{1} ; L_{2}[\right.$, then the receiving node assumes it is located in the same row/column as the sender and sets its corresponding coordinate as the same as the sender's one. Similarly, if the message is received with an RSSI within the range $\left[L_{2} ; L_{3}[\right.$, the receiving node is located one row/column away from the sender and it sets its corresponding coordinate to $X_{\text {sender }}+1$ (or $Y_{\text {sender }}+1$ ). Note that intervals must be leftclosed to avoid misinterpretation of the row/column to assign to a node. This procedure is performed by all nodes up to the $\left(N_{x}-\right.$ $1)^{\text {th }}$ column (resp. $\left(N_{y}-1\right)^{\text {th }}$ row). In all cases, a receiving node notifies the new coordinate to the second corresponding node on the same object. The procedure is summarized in Algorithm 1.

\subsection{Reducing the number of broadcasted messages}

One of the issues in most of the positioning algorithms is the large amount of messages exchanged between nodes. The consequences are, in general, higher energetic costs, collisions, and an overall decrease in protocol performance. In order to limit the number of messages used for positioning, and to prevent collisions, some actions are undertaken. First, since we assume that a broadcasted message can be heard as far as the $\left(N_{x}-1\right)^{\text {th }}$ column (resp. $\left(N_{y}-1\right)^{\text {th }}$ row) away, we set the restriction that only the nodes that are in the $\left(N_{x}-1\right)^{\text {th }}$ column (resp. $\left(N_{y}-1\right)^{t h}$ row $)$ rebroadcast the positioning messages. The total number of broadcasted messages, called $T$, is computed as:

$$
T=R\left\lceil\frac{C}{\left(N_{x}-1\right)}\right\rceil+C\left\lceil\frac{R}{\left(N_{y}-1\right)}\right\rceil, N_{x}, N_{y}>1
$$

\footnotetext{
${ }^{2}$ Since the signal propagation model in the $\mathrm{ZY}$ plane is similar to the one observed in the XY plane, it suffices to apply, in addition, the same algorithm in this new plan $(\mathrm{ZY})$ to get a three dimension positioning.
}

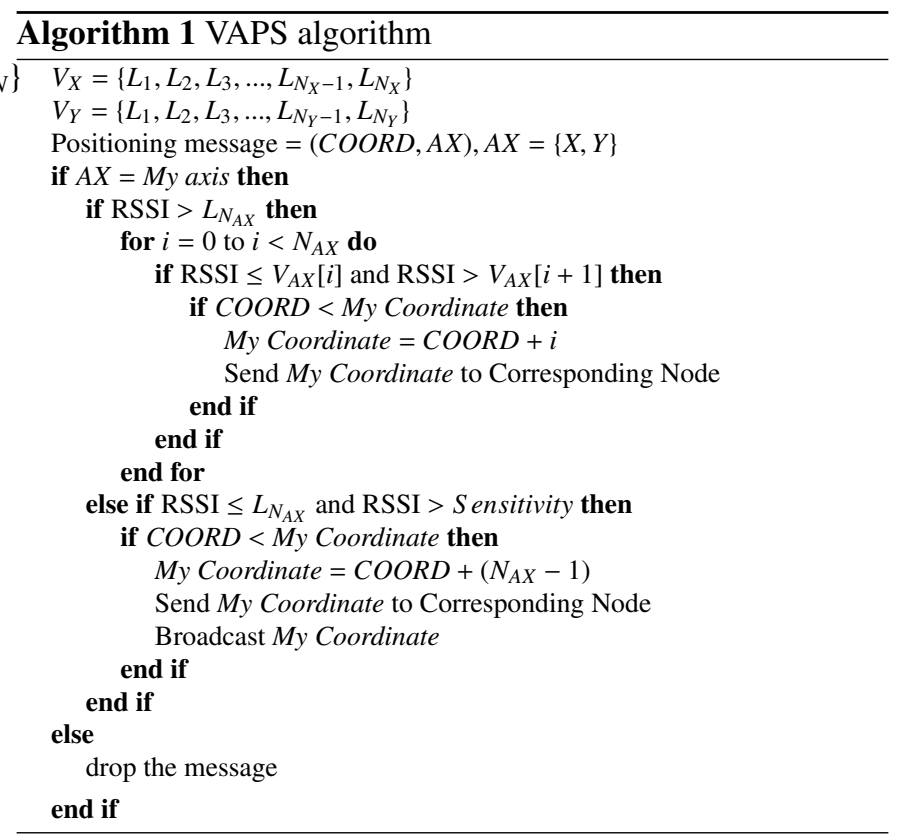

where $C$ is the number of columns and $R$ is the number of rows. Thus, the number of positioning messages broadcasted is reduced when compared to other traditional approaches where all nodes rebroadcast the positioning messages.

Second, besides triggering different start times for the positioning algorithm at each axis, VAPS includes a back-off procedure for rebroadcasting the positioning message reaching the $\left(N_{x}-1\right)^{t h}$ column (resp. $\left.\left(N_{y}-1\right)^{t h}\right)$. This procedure is similar to the collision avoidance procedure used in channel access algorithms. Upon reception of a positioning message, nodes in the $\left(N_{x}-1\right)^{\text {th }}$ column (resp. $\left(N_{y}-1\right)^{\text {th }}$ row) set up a timer with a random delay to retransmit the positioning message. The node whose timer expires first rebroadcasts the positioning message. The other nodes in the same row/column overhear this transmission and cancel their own transmission. More specifically, before canceling their transmission, these nodes first verify if the reception has been performed at the highest power and if the coordinate is the same as the one they have already stored.

It is worth mentioning that there is a tradeoff between the number of broadcasted messages and the accuracy and robustness of the system. Consider an ideal scenario where the area conditions allow the definition of $N \geq \max (C, R)$ distinguishable power levels. In such an ideal case, one positioning message per axis would be enough in order for every objects in the network to estimate its column and row number. Nevertheless, in this case, even mild changes in the propagation conditions may trigger positioning errors.

\subsection{Measuring the real distance between the nodes}

Since the dimensions of the objects and their arrangement are known beforehand, it is possible to easily and precisely calculate the distance between objects by using hop-count information. Let the objects be rectangular with height $a$ and length $b$. They are arranged on a grid with a vertical space $S_{v}$ and horizontal space $S_{h}$ between them as depicted in Fig. 9. The 


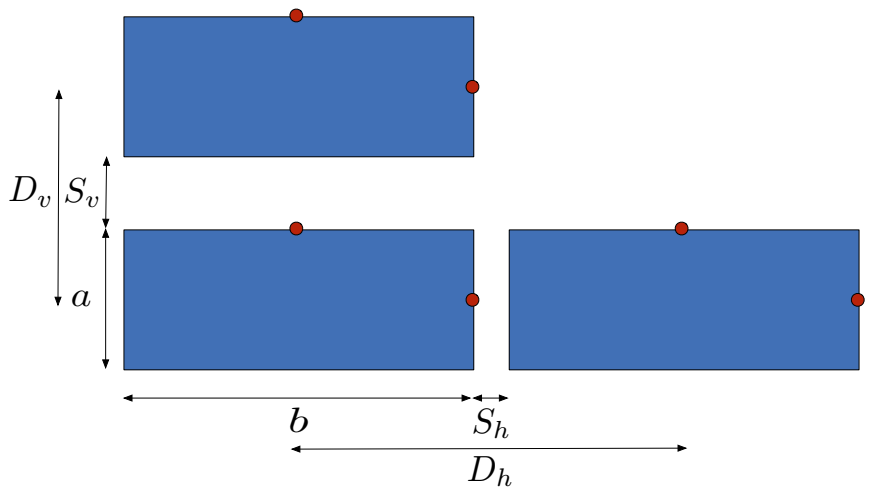

Figure 9: Calculation of the distance between containers.

vertical distance $D_{v}$ (resp. horizontal distance $D_{h}$ ) between the center of an object and the center of the next object is calculated as $D_{v}=\left(a+S_{v}\right)$ (resp. $\left.D_{h}=\left(b+S_{h}\right)\right)$. Therefore, the vertical (resp. horizontal) distance between an object, and another located $n$ hops away is $D_{v}=n\left(a+S_{v}\right)\left(\right.$ resp. $\left.D_{h}=n\left(b+S_{h}\right)\right)$.

\section{Evaluation}

To study the performance of VAPS, we evaluate it in a simulation environment adapted to our needs. Castalia [21, 22] is a Wireless Sensor Network (WSN) simulator based on the OMNeT++ Discrete Event Simulation System [23]. Castalia mainly focuses on providing a realistic wireless channel and radio model, with a realistic node behavior especially relating to access of the radio. The algorithms are written in $\mathrm{C}++$ and they are event driven, where an event can be an incoming message or a periodic timer. The OMNeT++ library controls the simulated time and the concurrent execution of the code running at each node. In the remainder of this section, we first discuss some specific implementation details of our simulations before presenting the results.

\subsection{Simulation settings}

In order to perform a set of simulations that closely represent real-life conditions, the interference model used in our simulations is assumed to be an additive model, in which all the transmissions heard by a receiver are added to the background noise as the interference level of the area. To accommodate the simulations to the particular propagation characteristics of the target scenario, we introduce the waveguide effect by setting signal propagation along an aisle between two rows of containers in an almost homogeneous way, with very low power attenuation. Note that this has a direct impact on communications, as it causes transmissions to be more prone to collide with each other.

One of the features of Castalia, besides providing a wireless channel propagation model, is that it allows the manual definition of connectivity between pairs of nodes, by specifying the received power level in $\mathrm{dBm}$ at one node, when the transmissions come from another node anywhere in the topology. We used this unique feature in order to manually define the particular connectivity between nodes according to the propagation characteristics of the scenario under consideration. According to the characterization we performed with WILDE, in the considered scenario there are three clearly distinguishable discrete power levels $(N=3)$; we set these to $L_{1}=0 \mathrm{dBm}, L_{2}=-$ $30 \mathrm{dBm}$, and $L_{3}=-60 \mathrm{dBm}$. Nevertheless, a manual definition of the reception level at one node would imply that all the received messages from another node arrive with the exact same power level each time. To introduce randomness in this process, and therefore obtain more realistic results, we add a random value extracted from a Gaussian distribution with zero mean and a parameterized standard deviation $\sigma$ to each reception power level.

Several simulation runs were conducted to evaluate the positioning error of VAPS. All data points shown on the curves represent an average over 50 runs within $95 \%$ confidence interval. The simulated topology contains 100 containers measuring $10 \times 4$ meters. This results in a total of 200 nodes, as each object is equipped with two sensors wired together (recall that we perform simulation only for the two dimensional case). We assume that all nodes have the same reception sensitivity ($95 \mathrm{dBm})$ and identical maximum transmission power $(10 \mathrm{dBm})$. Furthermore, as we focus on the positioning error, we assume that no packet loss occurs, however by using the back-off procedure in VAPS packet loss may occur rarely. The average positioning error is computed with the following equation, which is widely used in previous works:

$$
\operatorname{Err}_{\text {avg }}=\frac{\sum_{i=1}^{M} \sqrt{\left(x_{i}-x_{i}^{\prime}\right)^{2}+\left(y_{i}-y_{i}^{\prime}\right)^{2}}}{M},
$$

where $M$ is the number of nodes, $\left(x_{i}, y_{i}\right)$ is the real position of node $i$ and $\left(x_{i}^{\prime}, y_{i}^{\prime}\right)$ is the estimated position of node $i$.

As in a real marine port terminal, containers are placed following a grid pattern on a $122 \times 62$-meter field. The anchor object is placed at the bottom left corner of the field. All objects are 1-meter apart horizontally and 2-meters apart vertically. Consequently, the waveguide effect is more accentuated in the horizontal aisles than in the vertical aisles.

\subsection{Analysis of the retransmission at the $N^{\text {th }}$ level}

Since we assume that a broadcasted message can be heard as far as the $N^{\text {th }}$ row/column away, in our algorithm we set the restriction that only the nodes that are in the $N^{\text {th }}$ row/column rebroadcast the positioning messages. As one of the motivations for this choice is to reduce positioning errors induced by collisions, we evaluated the impact of this choice on the performance of the algorithm. We consider three different values of $N=\{1,2,3\}$.

Fig. 10 illustrates the average positioning error for retransmission at different aisles. Under ideal propagation conditions $(\sigma=0)$, when retransmitting at every aisle $(N=1)$, every two aisles $(N=2)$, and every three aisles $(N=3)$, the average positioning error is zero hop. In the presence of mild changes on propagation conditions $(\sigma=6)$, retransmitting at every aisle leads to higher positioning errors (about 0.4 hops), than retransmitting every two and every three aisles (close to zero). When 


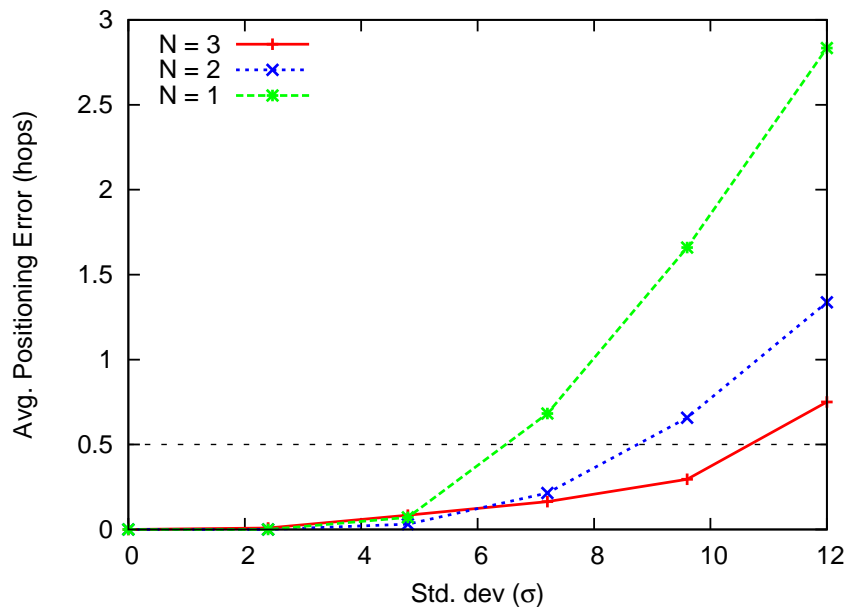

Figure 10: Average positioning error vs. Standard Deviation $\sigma$ for message retransmission at different aisles.

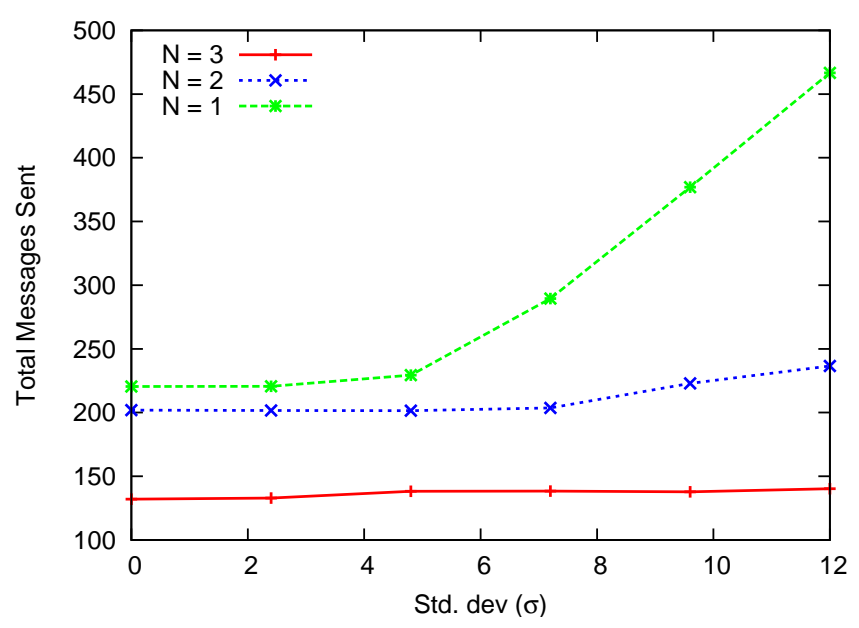

Figure 11: Total number of sent messages for message retransmission at different aisles.

there are considerable changes $(\sigma=12)$ on propagation conditions, when retransmitting at $N=1$ the average positioning error is 2.8 hops, at $N=2$ is 1.4 hops, and at $N=3$ is 0.8 hops. Clearly, by restricting the retransmissions to occur only at the last distinguishable level, the algorithm obtains a superior performance, since the possibility of collisions is reduced.

Another motivation behind the choice of retransmission at the $N^{\text {th }}$ row/column is to reduce the number of messages used for positioning proportionally to $1 / N$. This is what we observe in Fig. 11, which shows the total number of messages transmitted for each setting. When retransmission is done at each reception $(N=1)$, the total number of transmitted messages can reach 460 in the presence of considerable changes of propagation conditions. Retransmitting every two aisles results on a clear drop in the total number of messages to around 240. By setting the retransmissions at $N=3$, the total number of retransmissions is reduced to 140 . Reducing the total number of messages has a direct impact on the protocol costs in terms of control message overhead and energy.

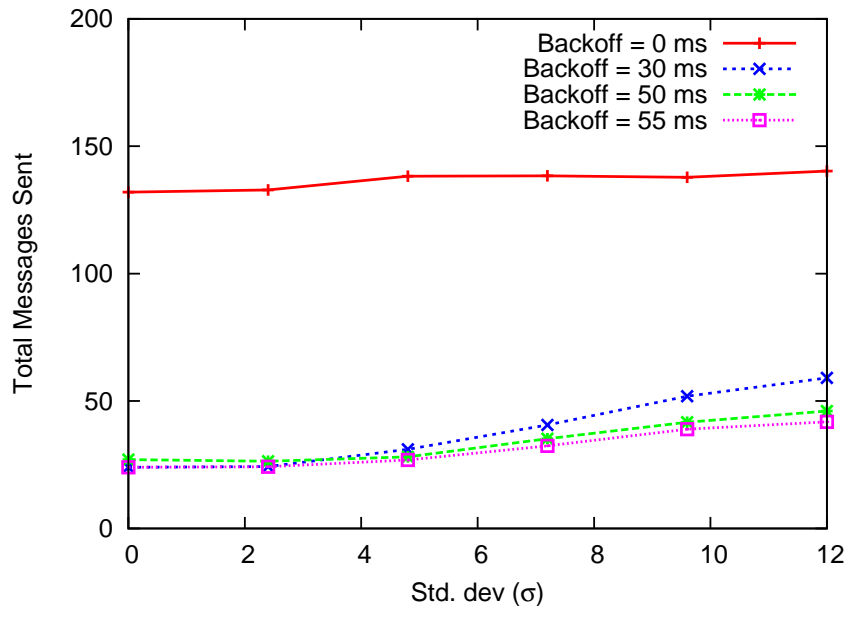

Figure 12: Total number of sent messages for different Back-off values.

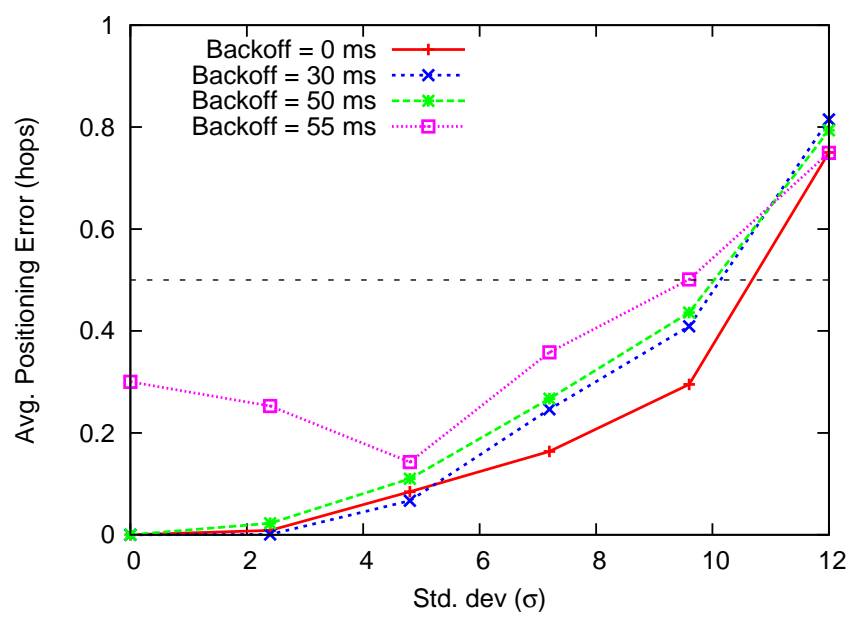

Figure 13: Average positioning error vs. Standard Deviation $\sigma$ for different Back-off values.

\subsection{Impact of the back-off procedure}

As previously stated, we also introduce a back-off procedure to to further improve the performance of the algorithm and to reduce the number of messages used for positioning. The idea is to take advantage of the waveguide effect present in the aisles between containers.

To choose an appropriate value for the back-off timer, we conducted a set of simulations. Fig. 12 shows the total number of transmitted messages as a function of the standard deviation $\sigma$. The duration of the back-off timer is varied from 0 to $55 \mathrm{~ms}$. As shown in this figure, without the back-off procedure, the total number of messages sent is about 130. This number is reduced down to 25 by increasing the back-off timer. When there are changes in the propagation conditions, the number of used messages increases. This is mainly due to nodes not being able to hear the transmission of the node whose timer expires first, and therefore triggering their own transmissions at the end of their timer.

Fig. 13 compares the average positioning error for different values of the back-off timer. In the figure, under ideal condi- 


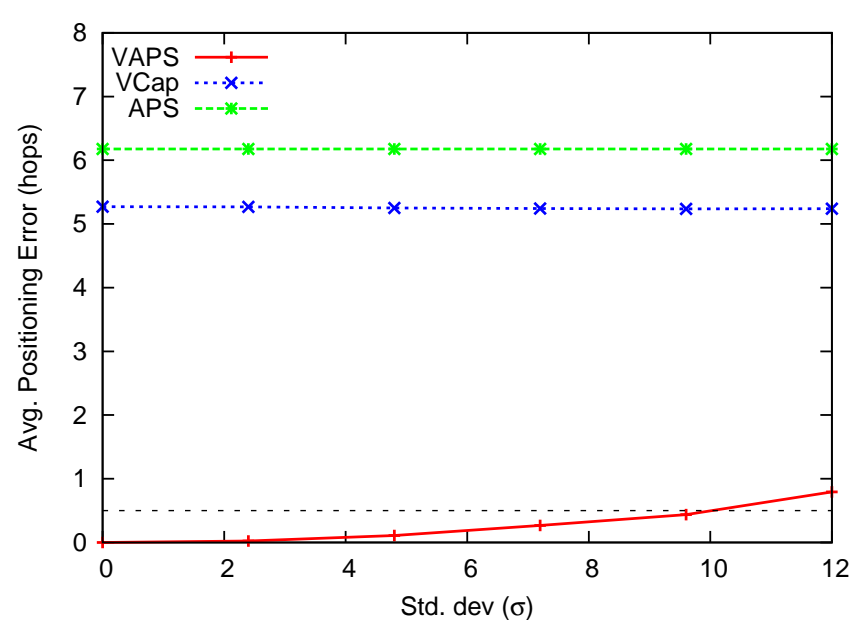

Figure 14: Average positioning error vs. standard deviation $\sigma$ for different approaches.

tions $(\sigma=0)$ the positioning error is zero for all back-off values except for $55 \mathrm{~ms}$, where it is around 0.3 hops. With strong variations in the RSSI $(\sigma=12)$, the average positioning error is about 0.7 hops when there is no back-off procedure, 0.8 for $30 \mathrm{~ms}$ and $50 \mathrm{~ms}$, and around 0.7 for $55 \mathrm{~ms}$. We can notice the fact that setting the back-off timer to a very large value (e.g., $55 \mathrm{~ms}$ ) induces errors even with mild variations in the propagation conditions. It suggests that there is a trade-off between the duration of the back-off timer and the accuracy of the system. The trade-off occurs because with large back-off timer values, the number of retransmitted messages is greatly reduced. This may lead to some cases where one or several nodes are not able to obtain their correct coordinates and thus the average positioning error of the system is increased. Since our system considers the volume and the spatial arrangement of the objects, when the average positioning error is below 0.5 hops (represented by a horizontal dotted line), it can be approximated to zero. Therefore, setting the back-off timer to $50 \mathrm{~ms}$ is a good candidate for the rest of the simulations.

\subsection{Comparison with other approaches}

We compare the performance of VAPS with two other algorithms (signal and topology based). The first algorithm, APS [6], uses only the RSSI and a free-space path loss model to estimate the distance from an object to a number of landmarks with known coordinates, and then trilateration to calculate the coordinates. A total of nine landmarks that broadcast periodic beacons are used to cover the whole area. The second algorithm is vCap [14], where the objects estimate their hop-count distances to three landmarks, and use this information directly as coordinates. Both APS and vCAP are explained in Section 7.

We first investigate the advantages of VAPS without applying the back-off procedure. The idea is to show that, even without using the back-off timer, VAPS outperforms the other approaches. Fig. 14 shows the average positioning error as a function of the standard deviation $\sigma$. The results show that, under ideal conditions $(\sigma=0)$, the positioning error is about 6

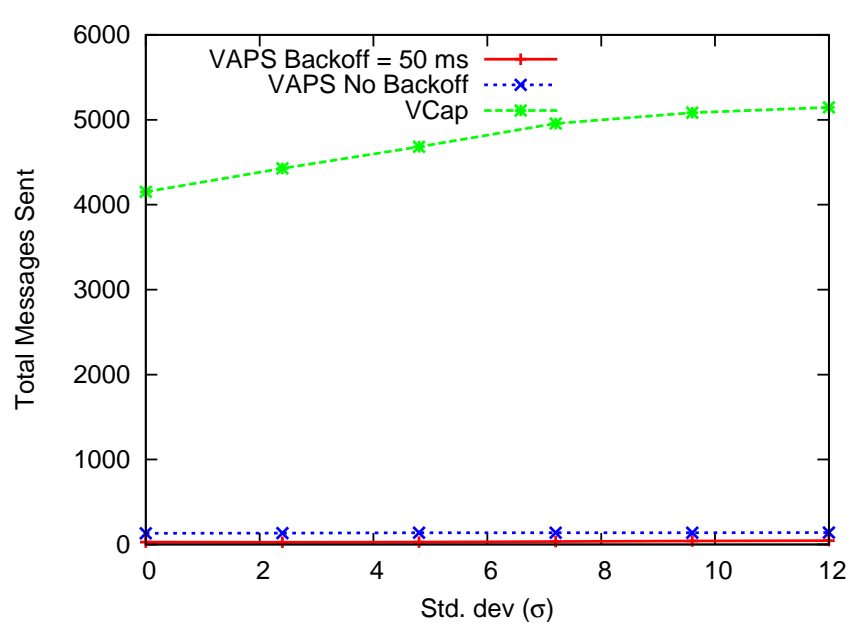

Figure 15: Total number of sent messages for different approaches.

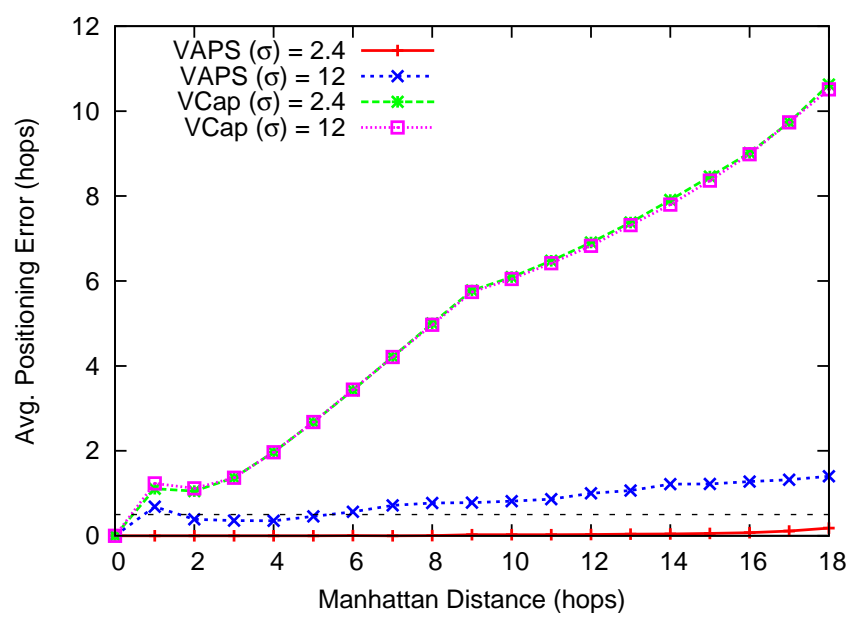

Figure 16: Average positioning error vs. Manhattan Distance for different approaches.

hops for the RSSI-based method, 5 hops for vCap, and 0 for VAPS. When there are strong variations in the RSSI at each node $(\sigma=12)$, the RSSI-based method and vCap obtain the same values as before, while VAPS achieves an average positioning error of 0.8 hops.

The comparison is further investigated in Fig. 15, which shows the total number of messages generated by vCap and VAPS. The results for APS are not shown since the number of messages generated by this algorithm is equal to the number of landmarks multiplied by the number of beacons they have sent during the simulation - i.e., there are no message retransmissions at the nodes. For vCap, the total number of transmitted messages is about 4,000 under ideal conditions, and increases up to 5,000 in the presence of considerable variations in the propagation conditions. VAPS dramatically reduces this number to around $130(\sigma=0)$ and to around 140 for $\sigma=12$ without the use of a back-off procedure. By using a back-off of $50 \mathrm{~ms}$, this value is further reduced to 25 under ideal conditions, and around 40 when the propagation conditions are highly variable. This reduction has a direct consequence on the protocol cost in 


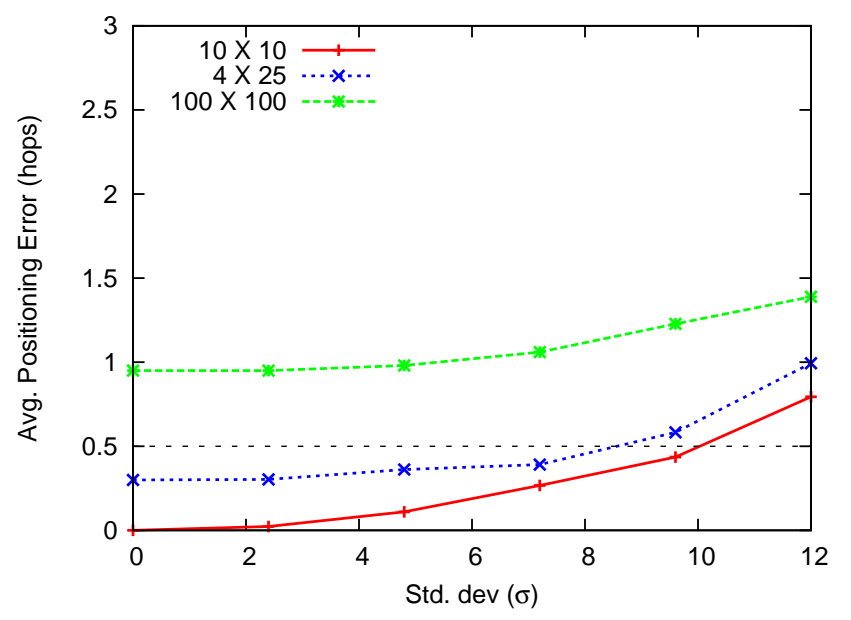

Figure 17: Average positioning error vs. standard deviation $\sigma$ for different topology sizes.

terms of control message overhead and energy.

To show how the positioning error is propagated away from the anchor object, we measure the average positioning error as a function of the Manhattan distance [24]. Again, the results for APS are not shown since, with this algorithm, the coordinates are calculated by each node with the information they receive from the landmarks, and therefore coordinate information is not propagated away from each landmark in a hop-by-hop fashion. Fig. 16 shows the results. We consider only the simulations where the standard deviation is 4 and 12, since these two values simulate respectively mild and considerable changes in the propagation conditions. In both cases, when the objects are relatively close to the anchor node ( 3 hops away), the average positioning error is around 1 hop for the hop-count-based method, and below 0.5 hops for VAPS. For VAPS, these errors can be approximated to zero, since it considers the volume and spatial constraints for the objects. For objects that are more than 14 hops away from the anchor node, the average positioning error for these standard deviations is about 8 hops for vCAP (for both $\tau=4$ and $\tau=12$ ), while it is 0 hops and 1 hop for VAPS, respectively. This figure shows that the capacity of VAPS to correct some degrees of positioning error lead to very low errors even in positioning far objects.

\subsection{Impact of the Size of the System}

In Fig. 17, we explore the performance of the algorithm in larger topologies. We chose two different sizes: $4 \times 25$ which contains the same number of nodes, but is larger towards the $\mathrm{x}$ axis, and $100 \times 100$ which contains 100 times more nodes. We deliberately chose not to include any modifications to the algorithm, such as changing the location of the anchor node or adding additional ones. As expected, for larger topologies, the performance of the algorithm is slightly degraded. Since the aisles are longer on the $\mathrm{x}$-axis, the nodes that are the farther away from a transmitting node will receive a more attenuated signal, which may cause a node to be unable to distinguish the row it is on, thus inducing positioning errors.

\section{Discussion}

Feasibility. It is clear that there is a class of deployment scenarios where signal propagation does not follow the traditional free space [20] or indoor [25, 26] propagation models. In these particular cases, the use of existing positioning methods (cf. Section 7) may lead to considerable positioning errors. The results shown in Section 5 highlight this fact. Consequently, existing positioning methods are simply unfeasible in such a deployment scenario. VAPS was designed to provide accurate positioning in cases where the spatial arrangement and the physical composition of the objects have a major impact on signal propagation. Along these lines, our algorithm proved to be superior to existing approaches.

Efficiency. In the scenario tackled in this paper, the choice of the number of levels, as well as their values, aimed at reducing the impact of changes in propagation condition changes on the accuracy of the algorithm. VAPS also relies on a back-off procedure and on limited retransmissions to reduce the number of messages used for positioning. Although this increases the probability of missing a message, the reduction in the number of positioning messages leads to significant improvement of the system's efficiency. The reduction of interfering collisions facilitates the computation of exact coordinates, while reducing the signaling cost of the algorithm. Indeed, since our algorithm is able to use less signaling traffic, it is less penalizing to concurrent applications.

Energy Consumption. VAPS was designed to work with wireless communication protocols adapted to embedded applications requiring low power consumption, such as IEEE 802.15.4. Moreover, by using fewer messages, there is a lower consumption of energy. As one of the main concerns when deploying wireless applications is the autonomy of the system, a preliminary task such as positioning should not consume the energetic resources of the nodes. VAPS reduces the energetic cost of the system, therefore allowing other applications to run longer.

Environment knowledge. One of the distinct features of our algorithm is its ability to map a number of clearly distinguishable discrete power levels into hop-counts. In order to accurately define the number of power levels to be used, and the range of values every level should represent, the propagation conditions must be studied and analyzed prior to launching the algorithm. Additionally, since our approach is addressed to a class of scenarios where wave propagation follows particular characteristics, it is clear that this study must be conducted for each scenario and will most likely lead to a different set of power levels each time. The careful definition of the number of power levels to use and their values are fundamental for the good operation of our approach. Also, as the dimensions of the objects and their specific arrangement are known beforehand, VAPS has the ability to correct positioning errors when they are below 0.5 hops. The conducted simulations showed that, even if the approach is sensible to changes in the propagation conditions, only considerable changes induce errors in the algorithm, and those errors are nonetheless under 1 hop. 
Open issues. Even though our approach was conceived to provide accurate positioning in real life deployment scenarios with particular wave propagation, it has been so far evaluated through simulations. Although these simulations were conducted using tools that closely reproduce the conditions found in a real life scenario, it is not possible to take into consideration all the factors that could affect the performance of the algorithm. Consequently, real wave propagation data obtained at a marine port terminal is needed to complement the data obtained from simulations. Additionally, a real life deployment is needed in order to evaluate the performance of the system and to introduce further improvements.

The results shown in Section 5 illustrate that VAPS cannot scale to infinity. It is expected, as waveguide are not perfect, and dispersion is expected. Nevertheless, even in larger topologies the performance of VAPS is superior to the performance of existing positioning algorithms. A future version of the algorithm should include the necessary adjustments (e.g., additional anchor nodes and additional retransmissions) in order to deal with the issues.

In this paper, we did not consider the case where one or more objects are missing, In this case, when the topology includes "holes", the propagation conditions would most certainly change in some areas, and would probably lead to inaccurate positioning. A future version of the algorithm should include a contention mechanism, where the system is able to detect one or several objects missing, and act accordingly in order to reduce the effects this particular situation might have on the performance of the algorithm.

VAPS does not consider cases where objects are able to move around and change positions. In these cases, positioning error will be induced by objects moving out of the coordinate where they were located initially, and propagating wrong coordinates to adjacent nodes. We also plan to include such a feature in a future version of the algorithm, by determining how often the algorithm should be re-run.

Our algorithm was conceived to position objects with a volume, and as such, in its design we take into account the threedimensional case. However, in order to simplify the analyses, and due to the limitations of the tools used for evaluating our approach, the description of the algorithm, as well as its evaluation, only include the two-dimensional case. Nonetheless, the extension of the algorithm to the three dimensional case is straightforward.

\section{Related work}

Ever since the appearance of the Global Positioning System (GPS) and the multi-billion dollar market it spawned, the positioning of objects has been a widely studied research subject. The existing positioning methods can be divided into two categories: signal-based methods, where the distance between a point and several reference points with known positions is estimated or calculated and then, by trilateration or other methods, the position of the point can be determined; and topology-based methods, where a node position is determined by using network topology metrics such as the hop-count between nodes.
Most of the current positioning techniques are based on physical measurements of signal propagation. Some methods use the RSSI in order to estimate the distance between a node and a reference point. RSSI is a measurement of the power of a received radio signal. Using this measurement and a propagation loss model, distance from a point in space to a radio source can be easily estimated. The RADAR Location System [5] uses RSSI information in order to locate and track a node inside a building. This system relies on an off-line data collection phase, where three base stations will record RSSI information from nodes in order to construct a map of the area. After this phase, a node is able to infer its position by comparing its RSSI measurements with the previously recorded data. Niculescu et al. propose Ad hoc Positioning System (APS) [6], which is a simplified version of GPS. To calculate its position, a node estimates its distance to a minimum of three landmarks by using RSSI or by using a distance vector algorithm if the node is multiple hops away from the landmark. Hop-TERRAIN [7] uses a similar approach to estimate the position of a node, which will then be iteratively refined to improve accuracy. Niculescu et al. propose in [29] a comprehensive survey on these techniques.

More recently, several research efforts have been oriented towards the use of topology metrics in order to determine the position of a node. GPS-Less [30] needs a limited number of nodes with known positions and overlapping regions of coverage to serve as reference points that periodically transmit beacon signals containing their position. Each node will infer its proximity to a set of reference points when the ratio of received to sent beacons metric surpasses a certain threshold. A node locates itself to the region where the coverage regions of these reference points intersect. In No-GEO [13], Rao et al. propose a system where the nodes are able to find their positions from the edge of the network towards the center of the network. Two designated bootstrap nodes broadcast beacons to the entire network. Nodes use their hop-count distances to one of the bootstrap nodes to determine whether they are perimeter nodes. Then every perimeter node broadcasts a message to the entire network, allowing other nodes to compute a vector containing their distance to all perimeter nodes. Using inter-perimeter distances, each node calculates normalized coordinates for both itself and the perimeter nodes by running a relaxation algorithm. With each iteration, a node will compute its position based in the position of its neighbors. The resulting position of a node is the average of its neighbors'. Caruso et al. propose vCap [14], a three-dimensional coordinate system based only in the hop-count distances between nodes. After network deployment, three nodes located on the edge of the network will be selected as anchors. These anchors will broadcast, one at a time, an election message to the entire network so that each node can calculate its own hop-count distance to each anchor. These distances will be directly used as three-dimensional coordinates. A similar approach, called GPS-Free-Free, is proposed by Benbadis et al [15]. GPS-Free-Free needs three landmark nodes. Each landmark will flood one at a time a message containing its identification and a hop-counter initially set to 0 . Upon reception of this message, each node will update its hop-count distance to each one of the landmarks and it will retransmit 
the received message with the hop-counter incremented by one. After the flooding phase, each node calculates both the position of each landmark and its own position in a two-dimensional space by trilateration. Another system proposed by Benbadis et al., Millipede [16], places landmarks at the perimeter of a network. Each node starts with a preprogrammed position and it will update it by retrieving the positions of its neighbors and calculating an average, through a relaxation algorithm similar to the one proposed in [13].

In summary, all of these methods assume that objects are just small points in space, without any dimensions whatsoever. As a consequence, there is no consideration of the effects that the volume and physical characteristics of an object and its spatial arrangement may have on signal propagation. It is therefore clear that, in certain deployment scenarios where signal propagation follows a particular path loss model (cf., Section 2), the limitations described in Section 3 apply to all of these approaches.

\section{Conclusion}

In this paper, we have advocated the fact that it is fundamental to consider spatial constraints in the design of positioning algorithms. In our work, new challenges arise from the waveguide/blocking effects induced by (metallic) containers in a harbor. These effects lead to a homogeneous signal propagation in the area. As existing RSSI-based positioning methods found in the literature do rely on signal attenuation, they lead to incorrect distance estimations. Similarly, traditional hop-count-based solutions also fail as all nodes in the same aisle are neighbors of each other.

To respond to these specificities, we have analyzed precisely the signal propagation model in an area with metallic objects. Using the result specifications of the environment, we introduced VAPS, a volume-aware positioning system that considers the volume and the physical characteristics of the containers when computing coordinates. VAPS takes advantage of the particular characteristics of the environment and relies on the definition of a number of discrete RSSI levels and their translation into hop-count distances. Through simulations, we observed that VAPS achieves an average position error of 0 , in scenarios where traditional approaches are unable to provide accurate positioning. Furthermore, by introducing features such as restricting message retransmissions and a randomized back-off procedure, the total number of transmitted messages is greatly reduced when compared to existing approaches, which implies lower costs both in terms of control overhead and energy.

\section{References}

[1] B. Hofmann-Wellenhof, H. Lichtenegger, J. Collins, Global Positioning System (GPS). Theory and practice, 5th Edition, Springer, Wien, 2001.

[2] Glonass.

URL http: //www.glonass-ianc.rsa.ru

[3] Galileo.

URL http://www.esa.int/esaNA/galileo.html
[4] B. Shaofeng, J. Jihang, F. Zhaobao, The beidou satellite positioning system and its positioning accuracy, Navigation 52 (3) (2005) 123-129.

[5] P. Bahl, V. N. Padmanabhan, RADAR: An In-Building RF-based User Location and Tracking System, in: IEEE Infocom, Vol. 2, Tel Aviv, Israel, 2000, pp. 775-784.

[6] D. Niculescu, B. Nath, Ad hoc positioning system, in: IEEE Globecom, no. 1, San Antonio, TX, USA, 2001, pp. 2926-2931.

[7] C. Savarese, J. M. Rabaey, K. Langendoen, Robust Positioning Algorithms for Distributed Ad-Hoc Wireless Sensor Networks, in: USENIX Annual Technical Conference, Philadelphia, PA, USA, 2002, pp. 317327.

[8] X. Ji, H. Zha, Sensor positioning in wireless ad-hoc sensor networks using multidimensional scaling, in: IEEE Infocom, no. 1, Hong Kong, PR China, 2004, pp. 2652-2661.

[9] C.-L. Wang, Y.-W. Hong, Y.-S. Dai., A decentralized positioning method for wireless sensor networks based on weighted interpolation, in: IEEE International Conference on Communications, Vol. 30, Glasgow, Scotland, 2007, pp. $3167-3172$.

[10] A. Savvides, H. Park, M. B. Srivastava, Dynamic fine-grained localization in ad-hoc networks of sensors, in: ACM Mobicom, Rome, Italy, 2001, pp. $166-179$.

[11] X. Cheng, A. Thaeler, G. Xue, D. Chen, TPS: A time-based positioning scheme for outdoor wireless sensor networks, in: IEEE Infocom, no. 1, Hong Kong, PR China, 2004, pp. 2685-2696.

[12] D. Niculescu, B. Nath, Ad hoc positioning system (APS) using AOA, in: IEEE Globecom, no. 1, San Francisco, CA, USA, 2003, pp. $1734-1743$.

[13] A. Rao, S. Ratnasamy, C. Papadimitriou, S. Shenker, I. Stoica, Geographic Routing without Location Information, in: ACM Mobicom, San Diego, CA, USA, 2003, pp. 96-108.

[14] A. Caruso, S. Chessa, S. De, A. Urpi, GPS free coordinate assignment and routing in wireless sensor networks, in: IEEE Infocom, no. 1, Miami, FL, USA, 2005, pp. 150-160.

[15] F. Benbadis, T. Friedman, M. Dias de Amorim, S. Fdida, GPS-Free-Free positioning system for sensor networks, in: International Conference on Wireless and Optical Communications Networks, Dubai, United Arab Emirates, 2005, pp. 541-545.

[16] F. Benbadis, J. Leguay, V. Borrel, M. Dias de Amorim, T. Friedman, Millipede: a rollerblade positioning system, in: ACM Wintech, Los Angeles, CA, USA, 2006, pp. 117-118.

[17] Projet surveiller et prévenir, http://svp.irisa.fr.

[18] WILDE : Wireless LAN Design TOOL.

URL http://perso.citi.insa-lyon.fr/jmgorce/ recherche_engl.htm

[19] J.-M. Gorce, K. Jaffres-Runser, G. de la Roche, Deterministic Approach for Fast Simulations of Indoor Radio Wave Propagation, IEEE Transactions on Antennas and Propagation 55 (3) (2007) 938-948.

[20] C. A. Balanis, Antena theory: analysis and design, 3rd Edition, Wiley, Hoboken, N.J., 2005.

[21] Castalia. A simulator for WSNs. URL http: //castalia.npc.nicta.cm.au

[22] H. N. Pham, D. Pediaditakis, A. Boulis, From Simulation to Real Deployments in WSN and Back, in: IEEE International Symposium on a World of Wireless Mobile and Multimedia Networks, Helsinki, Finland, 2007, pp. 1-6.

[23] A. Varga, The OMNeT++ discrete event simulation system, in: Proceedings of the European Simulation Multiconference, Prague, Czech Republic, 2001, pp. 319-324.

[24] E. F. Krause, Taxicab geometry: an adventure in non-euclidean geometry, Dover, New York, 1986.

[25] S. Y. Seidel, T. S. Rapport, 914 MHz path loss prediction Model for Indoor Wireless Communications in Multi-floored buildings, IEEE Transactions on Antennas and Propagation Propagation 40 (2) (1992) 207 217.

[26] International Telecommunication Union, Recommendation ITU-R P.1238-4 - Propagation data and prediction methods for the planning of indoor radio communication systems and the radio local area networks in the frequency range $900 \mathrm{MHz}$ to $100 \mathrm{GHz}$

[27] I. Borg, P. Groenen, Modern multidimensional scaling: theory and applications, Springer, New York, 2005.

[28] E. L. Lehmann, G. Casella, Theory of Point Estimation, 2nd Edition, Springer, New York, 1998. 
[29] D. Niculescu, B. Nath, Position and orientation in ad hoc networks, Ad Hoc Networks 2 (2) (2004) 133-151.

[30] N. Bulusu, J. Heidemann, D. Estrin, GPS-less low-cost outdoor localization for very small devices, IEEE Personal Communications 7 (5) (2000) 28-34. 